P-380 PERSONAL EXPOSURE TO OCCUPATIONAL RADIOFREQUENCY ELECTROMAGNETIC FIELDS: RESULTS FROM A PILOT

${ }^{1}$ Maxime Turuban, Hans Kromhout, Javier Vila, Ghislaine Bouvier, Elisabeth Cardis, Michelle Turner, José Antonio Alcolea. 'ISGlobal, Spain

\subsection{6/OEM-2021-EPI.301}

Background A Job-Exposure-Matrix (JEM) for occupational exposure to radiofrequency (RF) electromagnetic field (EMF) was previously developed based on source measurements and not on personal measurement data. In the framework of an international study aimed at validating and enriching the JEM, we conducted a pilot study of personal measurements of occupational exposure to RF fields in a rehabilitation department of a Spanish hospital among various groups of workers in order to develop a protocol for the main field study in three countries.

Methods We previously identified and prioritized the types of occupations to be measured based on their presumed levels of exposure to RF fields from the RF-JEM and based on expert judgment. A screening questionnaire was used to identify potential participants, based on their job titles and reported sources of RF exposure. Consequently, personal full-shift measurements were collected. In addition, a worker's dairy collecting information on occupational-specific sources of RF used during the measurement day, the use of personal mobile phones and general occupational data was filled in by every participant at the end of the shift.

Results A total of 16 workers (cleaning staff, receptionists and physiotherapists) participated in the Spanish pilot test. Overall geometric and arithmetic personal exposure levels were low; however, peaks of exposure to electric (>125 $\mathrm{V} / \mathrm{m})$ and magnetic fields $(>0,55 \mathrm{~A} / \mathrm{m})$ exceeding the ICNIRP 1998 occupational standards at the frequency of $27,12 \mathrm{MHz}$ were observed for a small number of participants, though peak duration was brief and their frequency scarce.

Conclusion We performed personal measurements of RF occupational exposure in a pilot study within the framework of an international study. Measurements will be performed across a wide range of occupations in Spain, France and the Netherlands and will be used to update and enhance an existing RF-JEM to be use in occupational epidemiological research.

\section{P-381 COMPARISON OF URINE N-METHYLFORMAMIDE AMONG THE MIGRANT AND NATIVE WORKERS AT RISK OF EXPOSURE TO N, N-DIMETHYLFORMAMIDE IN MANUFACTURING INDUSTRIES IN THE REPUBLIC OF KOREA (2012-2019)}

'LEE Kyung-Eun, Park Ga-young. 'Occupational Safety and Health Research Institute, Republic of Korea, and Korea Occupational Safety and Health Agency, Republic of Korea

\subsection{6/OEM-2021-EPI.302}

Introduction N-N-dimethylformamide (DMF) which could induce to liver toxicity was the most common chemicals attributing $27.3 \%$ of the intoxication accidents of which $33 \%$ involved the migrant workers at workplace. Occupational health studies of the migrant workers at risk of exposure to DMF are rare despite their social vulnerabilities due to the lack of information owing to the deficiencies in the national monitoring system.

Objectives The aim of this study is to assess the urine concentration of $\mathrm{N}$-methylformamide, a biological monitoring index (BEI) of DMF, among the migrant workers at risk of exposure to DMF by comparing with the native workers in manufacturing industry.

Methods This cross-sectional study was designed to target employees aged 20 and more, who were eligible to participate in the special health examination for DMF exposure in 20142019. Data were obtained from the workers' special health examination (WSHE) and Work Environment Measurement (WEM) database of 2014-2019, managed by the KOSHA. We assessed the fundamental characteristics known to affect DMF exposure and its metabolism in human body including demographics (sex, age), companies where the subjects are employed (number of workers, types of industries), and DMF exposure factor (Time weighted average(TWA), years of employment). The Mann-Whitney U test and Pearson's chisquared test were used to compare the baseline characteristics between the native and migrant workers.

Results $60.6 \%$ of the migrant workers exposed to DMF were employed in small and medium sized company, while 19.6\% of the native workers were. $21.6 \%$ of the migrant workers were exposed to TWA of DMF exceeding Threshold Limit Values, which significantly high compared with the native workers $(6.3 \%)$. The mean concentration of urine $\mathrm{N}$-methylformamide in the migrant workers $(1.37 \mathrm{mg} / \mathrm{L})$ was significantly higher than in the native workers $(0.47 \mathrm{mg} / \mathrm{L}, \mathrm{p}<0$ $.001)$.

Conclusions The migrant workers at risk of DMF exposure in manufacture industries were in highly vulnerable environment at workplace.

\section{P-386 HEALTH SURVEILLANCE OF THE WORKPLACE MEDICAL SERVICES IN ECUADOR}

${ }^{1}$ Fernando Carpio, Altacilio Nunes. 'PUCE-UCE-USP, Ecuador

\subsection{6/OEM-2021-EPI.303}

Introduction Thanks to ILO Recommendation 112, companies with more than 100 workers must carry out pre-occupational, periodic and retirement medical evaluations. A study of these services has not yet been carried out in Ecuador. There is an underreporting of occupational diseases.

Objectives 1. To know the health prevention activities of the occupational medical services 2 . To know the opinion of the workers about the work of these services.

Methods A representative sample of company doctors belonging to the National Association was obtained. An anonymous questionnaire was administered to 64 doctors, with 42 questions about the characteristics of the company and the workforce, the equipment, the medical evaluations, the health promotion activities they carry out and about professional secrecy. Another survey was carried out on union organizations of 21 companies on similar aspects.

Results The incidence of occupational accidents was $14.34 \mathrm{x}$ 1000. The prevalence of occupational diseases was $1.01 \mathrm{x}$ 